\title{
Distinct and common cortical activations for multimodal semantic categories
}

\author{
R. F. GOLDBERG \\ University of Pennsylvania, Philadelphia, Pennsylvania \\ and Center for Cognitive Neuroscience, Philadelphia, Pennsylvania \\ and \\ C. A. PERFETTI and W. SCHNEIDER \\ University of Pittsburgh, Pittsburgh, Pennsylvania \\ and Center for the Neural Basis of Cognition, Pittsburgh, Pennsylvania
}

\begin{abstract}
If semantic representations are based on particular types of perceptual features, then category knowledge that arises from multimodal sensory experiences should rely on distinct and common sensory brain regions depending on the features involved. Using a similarity-based generation-andcomparison task, we found that semantic categories activated cortical areas associated with taste and smell, biological motion, and visual processing. Fruit names specifically activated medial orbitofrontal regions associated with taste and smell. Labels for body parts and clothing activated lateral temporal occipitoparietal areas associated with perceiving the human body. More generally, visually biased categories activated ventral temporal regions typically ascribed to visual object recognition, whereas functional categories activated lateral frontotemporal areas previously associated with the representation of usage properties. These results indicate that semantic categories that are distinguished by particular perceptual properties rely on distinct cortical regions, whereas semantic categories that rely on similar types of features depend on common brain areas.
\end{abstract}

Concepts are considered to be the building blocks of human higher order cognition (Margolis \& Laurence, 1999). Yet, theories differ according to how these symbolic representations are instantiated within the brain. The amodal characteristics of word meaning (Landauer \& Dumais, 1997) imply that knowledge is stored independently of perceptual experiences. Mounting evidence, however, suggests that concepts depend upon cortical regions typically ascribed to sensory input (Martin \& Chao, 2001). This embodiment of semantic representations through perceptual mechanisms can explain how word meanings necessarily draw upon sensory experiences of the referenced objects (Barsalou, 1999). Yet, theories of semantic knowledge differ according to the level at which object categories are said to rely on particular brain regions. These differences concern how semantic information is organized: by the sensory features of items (Martin \& Chao, 2001), by innate domain-based mechanisms (Caramazza \& Shelton, 1998), or on the basis of a uniform distribution (Forde \& Humphreys, 2002; Tyler \& Moss, 2001). The resolution of this debate is critical to solving how conceptual understanding is acquired, maintained, and even hindered by the supporting neural circuitry.

We thank I. Suzuki for her invaluable assistance and J. Chein, J. Fiez, P. Janata, J. McClelland, C. Olson, S. Thompson-Schill, and D. Tranel for their advice. We gratefully acknowledge support from the National Science Foundation. Send correspondence to R. F. Goldberg, Center for Cognitive Neuroscience, 3720 Walnut Street, Philadelphia, PA 19104 (e-mail: robg@psych.upenn.edu).
The neuropsychological literature is filled with case studies of individuals who demonstrate a selective loss of semantic memory (for recent reviews, see Capitani, Laiacona, Mahon, \& Caramazza, 2003; Caramazza \& Shelton, 1998; Saffran \& Schwartz, 1994), even though such cases are relatively rare (Coltheart, 2001). A distinction between natural kinds and artifacts has been the one most frequently reported among patients with semantic memory impairments (Mummery, Patterson, Hodges, \& Price, 1998). Damage localized to the inferomedial temporal cortex, usually resulting from encephalitis due to the herpes simplex virus, has been associated with impaired performance for natural kinds (e.g., animals, fruits, and vegetables) in comparison with a sparing of artifact (e.g., tools, household objects, or vehicles) knowledge (Gainotti \& Silveri, 1996; Hart \& Gordon, 1992). The reverse pattern of category-specific deficits (i.e., artifacts impaired in comparison with preservation of natural kind knowledge), usually caused by damage to frontoparietal areas, has also been found (Hills \& Caramazza, 1991; Sacchett \& Humphreys, 1992; Warrington \& McCarthy, 1987) - though significantly less often than selective impairments for natural kinds (Capitani et al., 2003). In sum, this double dissociation has led to the inference that semantic knowledge is organized on some level by category-specific information.

In the face of these category-specific deficits and more diffuse semantic disturbances when stimulus factors are rigorously controlled, a number of proposals have been advanced to explain the natural kind-artifact dissociation found among patients. The most widely accepted view assumes that there are substantial differences in the saliency 
of semantic features for particular categories (Mummery et al., 1998) and that the neural instantiation of these features is biased to higher order cortical areas that are mostly responsible for encoding them (e.g., visual features in extrastriate regions). The assumption is that natural kinds are distinguished from each other primarily by their visual properties, whereas artifacts are differentiated more by functional features. These category-specific deficits suggest, therefore, that semantic representations rely on dissociable sensory brain regions depending on the constituent features involved (Warrington \& Shallice, 1984). Patients with such deficits show a disproportionate impairment for recognizing items within some object categories, whereas other categories are preserved (Capitani et al., 2003). These case studies suggest that natural kind categories, such as animals and fruits, tend to rely on visual areas in the temporal cortex (Gainotti \& Silveri, 1996) to distinguish near associates in conceptual space. In contrast, categories of manipulable objects, such as tools and body parts, are thought to preferentially rely on function-biased areas in the lateral temporal and frontal cortex (Sacchett \& Humphreys, 1992). These results suggest that category-specific deficits arise from how different types of features and the categories that tend to rely on them are localized to distinct brain regions, even though controversy surrounding this interpretation remains (Caramazza \& Mahon, 2003; Martin \& Caramazza, 2003).

Recent neuroimaging studies provide converging support for the view that the features of items - not categories, per se - are represented by disparate brain regions. Martin and colleagues (for a recent review, see Martin \& Chao, 2001) have shown across a number of studies that visual properties and the semantic categories that preferentially rely on them (e.g., animals) tend to activate visual object recognition areas in the ventral temporal cortex. In contrast, action properties associated with manipulating an object tend to activate premotor and lateral temporal brain regions, providing converging support for the locations of category-specific properties of artifact categories (e.g., tools). These results argue for a distribution of object knowledge based on how cortical regions involved in encoding sensory features are used to discriminate among similar members of the same semantic category and to separate superordinate categories based on the collection of associated sensory experiences. For example, tigers are distinguished from leopards by the presence or absence of visual features such as stripes or spots. In contrast, artifacts are distinguished more by their functional attributes. That is, the same object may be called a jar, a vase, or a glass, on the basis of how it is used. Different types of properties and the cortical regions supporting them may therefore distinguish one semantic category from another, whereas semantic categories that rely on similar types of properties are likely to depend on common brain regions. For instance, Mummery and colleagues (Mummery, Patterson, Hodges, \& Wise, 1996) found greater activation bilaterally in the anteromedial temporal lobe for natural kind categories, whereas manipulable objects activated the posterior inferolateral temporal cortex, generally supporting the dissociation of these broad classes found in the lesion literature. Futhermore, studies on activation differences between animals and artifacts have been replicated (Martin, Wiggs, Ungerleider, \& Haxby, 1996; Perani et al., 1995), and their results demonstrated that left occipitotemporal regions in the ventral visual stream are more active for animals, and left prefrontal and premotor regions are more active for tools. These findings support the predictions of specific locations from the case studies.

These neuropsychological and neuroimaging findings may be interpreted more broadly in support of perceptual symbol theories, which presuppose that sensory experiences of objects support symbolic representations of them. Barsalou (1999) suggests that linguistic representations have their basis in sensory mechanisms in which the recollection of object properties involves perceptual simulations of the objects themselves. For instance, participants are faster to respond to properties that are perceptually-not conceptually - similar to those previously verified (Solomon \& Barsalou, 2001) and to pictures that match a described orientation of an item (Stanfield \& Zwaan, 2001). The cortical organization of semantic knowledge, therefore, may be determined by the topography of perceptual representations (Simmons \& Barsalou, 2003).

We examined the hypothesis that semantic representations for object categories are represented in multiple cortical areas, depending on the roles of specialized sensory mechanisms in encoding perceptual experiences with the associated objects. If the constituent features of items can predict how semantic knowledge is instantiated within the cortex, semantic categories should dissociate when the perceptual properties differ, whereas common brain regions should support superordinate categories that tend to rely on common types of features. Given the central role of feature similarity in behavioral research (see, e.g., Rosch, Mervis, Gray, Johnson, \& Boyes-Braem, 1976), we required participants to produce items and choose between alternatives on the basis of the judged similarity among individual word referents. Participants were asked to covertly generate the most similar item they could think of in relation to a target item (e.g., "What is the most similar item to peach?") and were then asked to choose one of two alternatives (e.g., nectarine or pear) that was the most similar to the one they generated. We intentionally included items with very similar but often low lexical familiarity (e.g., kiwi) to induce careful evaluation of each item and its related properties. This manipulation thus required between-items comparisons rather than either an explicit evaluation of the superordinate category or matching based on specific features, both of which are likely to evoke more analytic processing instead of the intended commonalities and differences between items and their associated classes. With this design, we expected multimodal category differences and the brain regions underlying them to emerge from participants' implicit knowledge of object similarity.

To investigate the cortical representation of object categories, we used functional magnetic resonance imaging (fMRI) to scan participants while they generated and compared items from the judged similarity to a target. In a blocked design, items were drawn from superordinate categories that differed according to their predicted constituent 
features. Category contrasts were expected to show unique regions of activation if the constituent feature types differed, whereas the contrasts were expected to demonstrate common areas of activation if categories relied on similar feature types. We hypothesized that these categorical similarities and differences would reflect the sensory processing associated with some object classes but not others. For instance, categories that were assumed to preferentially depend on visual properties were expected to activate common regions of the extrastriate cortex, as indicated by deficits for natural kinds with ventral temporal damage (Gainotti \& Silveri, 1996; Warrington \& Shallice, 1984) and the selective activation of ventral temporal regions when properties of object form knowledge are considered (Martin \& Chao, 2001). As supported by evidence from lesion (Sacchett \& Humphreys, 1992; Warrington \& McCarthy, 1987) and neuroimaging (Beauchamp, Lee, Haxby, \& Martin, 2002; Downing, Jiang, Shuman, \& Kanwisher, 2001) studies, judgments of body parts (but not the other tested categories) were expected to specifically activate distinct cortical regions associated with perception of the human form. By contrast, comparisons among fruit names were expected to elicit activation in regions involved in sensory processing for these items but not for any of the other categories.

\section{METHOD}

\section{Participants}

Sixteen right-handed native English speakers were recruited from the University of Pittsburgh community (mean age $=23 ; S D=$ 1.61). Three participants were removed from this sample because of excessive head motion ( $>3 \mathrm{~mm}$ ) during the scanning session. Therefore, a total of 13 participants were included in the data analyses. The protocol for this study was approved by the Institutional Review Board at the University of Pittsburgh, and informed consent was obtained from all participants.

\section{Stimuli and Design}

Word stimuli were drawn from one of four semantic categories: birds, fruit, body parts, and clothing, on the basis of the presumed role of multimodal feature sets in each. All presented word items across the tested semantic categories were matched for letter length, number of syllables, and lexical familiarity. Lexical familiarity was derived from response times and accuracy data for each item when presented in a lexical decision experiment to a separate set of participants drawn from the same population as the neuroimaging sample. This effort aimed to determine a direct empirical measure for how well word forms are known, in comparison with text-driven frequency estimates (Francis \& Kučera, 1982), which are susceptible to corpora selection bias and inconsistencies between how words are used in speech and in print. We transformed the lexical decision data of about 90 participants for over 600 words into a standardized $z$-space based on mean and variance measures for response time and accuracy for each item across the sample (Goldberg, 2006). This index, therefore, reflects the ease with which the participants ascribed lexical status (e.g., algae $=-1.15$, whereas apple $=+1.05$ ), with the most positive scores representing items that are, on average, responded to very quickly and accurately in the lexical decision task.

This study developed a similarity-based paradigm that required the participants to generate and compare word items on the basis of their shared relations. Each 6-sec trial (see Figure 1) began with the presentation of a fixation cross for $500 \mathrm{msec}$ and a target item for $2 \mathrm{sec}$, followed by another 2-sec period for the participants to generate the most similar item they could think of in relation to the target. A forced choice comparison decision was presented for $1.5 \mathrm{sec}$, and the participants were instructed to choose the item most similar to the one they had generated. Four trials within each category were presented consecutively in a blocked design, with each category block randomly presented six times across the experiment. Thirty items were presented within each category across 24 trials. No item was presented as a target more than once, and no forced choice pair was repeated.

The participants were not informed of the categorical grouping of items; rather, they were told only to consider the overall similarity between items. We expected that this manipulation would drive activation associated with the relationship between items, in contrast to passive decisions. This design allowed for the examination of regional activations specific to and shared between the tested categories, without an explicit instruction to consider the superordinate class. The categories were chosen to maximize between-category effects and to test differences between those categories that rely on visual information (i.e., birds and fruits), and those that rely on functional differences (i.e., body parts and clothing), as indicated in prior case studies with patients (for a thorough review, see Capitani et al., 2003). Therefore, this similarity-based generate-and-compare paradigm allowed us to analyze the distinct and common cortical regions associated with increased activation across the set of tested categories.

Prior to the scanning session, the participants were trained on the paradigm by using categorical stimuli (e.g., insects) that were not presented in the neuroimaging session. This training aimed to familiarize the participants with the task as well as to monitor and reinforce the goal of producing responses based on the overall similarity of items. In contrast to the covert generation utilized during the experiment, upon presentation of the target stimulus, the participants were asked to overtly generate the most similar item they could think of (e.g., tick $\rightarrow$ "gnat") so that feedback could be given if generated items were not similar to what the item represented. The participants were encouraged to generate more similar items when their overt responses were either too general (e.g., "bug") or when items were associated with but not similar to the target (e.g., "dog"). They were encouraged to continue generating items until they found what they

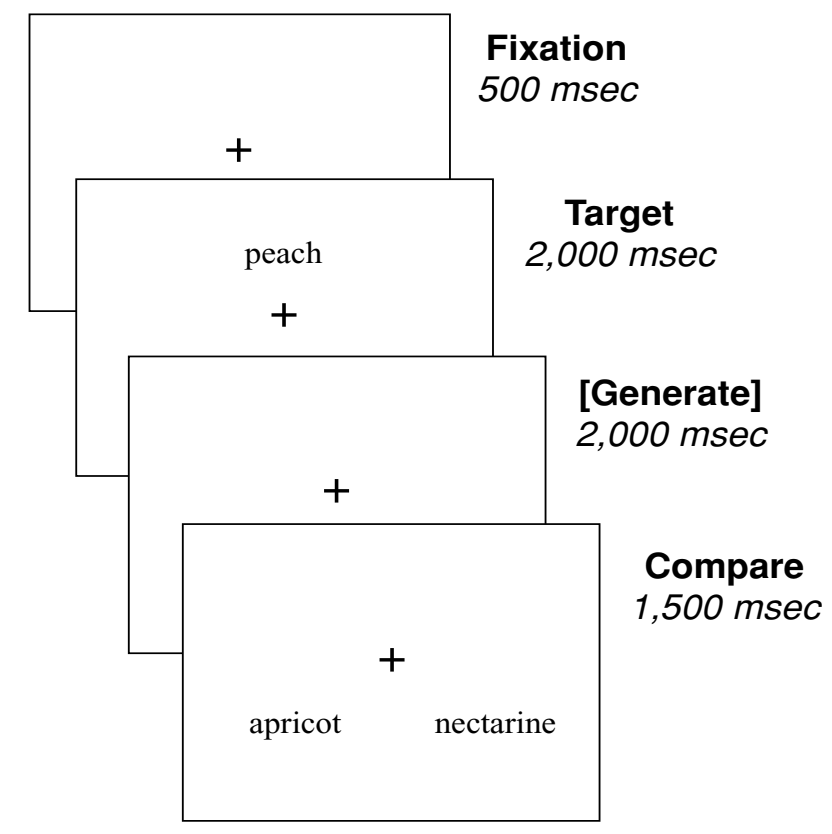

Figure 1. The paradigm was designed to elicit between-items similarity comparisons. In each trial, participants were asked to covertly generate the most similar item they could think of in response to a target word. They were then asked to compare two alternatives and choose the item most similar to the one they generated. 
Table 1 Areas Showing Category-Specific Activation

\begin{tabular}{|c|c|c|c|c|c|c|c|}
\hline \multirow[b]{2}{*}{ Category } & \multirow[b]{2}{*}{ Region } & \multirow[b]{2}{*}{$\mathrm{BA}$} & \multicolumn{3}{|c|}{$\begin{array}{c}\text { Talairach } \\
\text { Coordinates }\end{array}$} & \multirow{2}{*}{$\begin{array}{l}\text { Spatial } \\
\text { Extent } \\
\left(3-\mathrm{mm}^{3}\right. \\
\text { voxels })\end{array}$} & \multirow[b]{2}{*}{$t$ Value } \\
\hline & & & $x$ & $y$ & $z$ & & \\
\hline Birds & Left ventral temporal cortex & $19 / 36 / 37$ & -24 & -40 & -14 & 62 & 3.560 \\
\hline Body parts & $\begin{array}{l}\text { Right inferior frontal cortex } \\
\text { Left temporal occipitoparietal } \\
\text { Left inferior frontal cortex } \\
\text { Left middle frontal cortex }\end{array}$ & $\begin{array}{r}44 / 45 / 47 \\
22 / 37 / 39 \\
44 / 45 / 47 \\
6 / 9\end{array}$ & $\begin{array}{r}40 \\
-45 \\
-41 \\
-36\end{array}$ & $\begin{array}{r}16 \\
-60 \\
15 \\
7\end{array}$ & $\begin{array}{r}-4 \\
15 \\
11 \\
33\end{array}$ & $\begin{array}{r}214 \\
1,050 \\
379 \\
8\end{array}$ & $\begin{array}{l}3.589 \\
4.225 \\
3.707 \\
3.306\end{array}$ \\
\hline Fruits & $\begin{array}{l}\text { Right orbitofrontal cortex } \\
\text { Left orbitofrontal cortex } \\
\text { Left superior frontal gyrus } \\
\text { Left fusiform gyrus } \\
\text { Left middle frontal gyrus }\end{array}$ & $\begin{array}{r}11 / 12 \\
11 / 12 \\
10 \\
37 \\
10 / 46\end{array}$ & $\begin{array}{r}22 \\
-19 \\
-19 \\
-30 \\
-40\end{array}$ & $\begin{array}{r}36 \\
41 \\
52 \\
-50 \\
39\end{array}$ & $\begin{array}{r}-3 \\
-3 \\
17 \\
-12 \\
17\end{array}$ & $\begin{array}{r}74 \\
109 \\
72 \\
29 \\
39\end{array}$ & $\begin{array}{l}3.939 \\
3.997 \\
3.515 \\
3.672 \\
3.381\end{array}$ \\
\hline
\end{tabular}

thought was the best item or until the forced choice pair appeared. When the comparison choice was presented (e.g., flea or ant), the participants were asked to choose the item most similar to the one that they had generated. They were not instructed to consider the superordinate category to which the items belonged or to use an imagery strategy. Their responses, rather, were expected to correspond only to judgments of interitem similarity relations.

In visuomotor control trials, the participants were presented with a target stimulus consisting of a string of uniform symbolic characters (e.g., \#\#\#\#). During the generation phase, they were asked to name the symbols, overtly during the training session and covertly during scanning. At the choice point, the participants were asked to indicate the visual stimulus that they had named (e.g., \#\#\#\#\# or +++++ ).

\section{Data Acquisition}

Anatomical and functional images were collected on a full-body Signa 3 Tesla scanner (General Electric, Milwaukee, WI). The scanning session began with the acquisition of structural images that included scout, high-resolution SPGR volume $(256 \times 256$ matrix, one hundred twenty-four 1.2-mm sagittal slices), and T1-weighted $(128 \times 128$ matrix, thirty $3.0-\mathrm{mm}$ transverse slices with no gap) sequences. T $2 *$-weighted functional images $(\mathrm{TR}=3,000, \mathrm{TE}=$ 30; $64 \times 64$ matrix, thirty 3.0-mm transverse slices with no gap), aligned to the inferior aspect of the temporal lobes, were collected while the participants performed the task. Stimuli were presented and responses were collected using the E-Prime software package (Psychology Software Tools, Inc.).

\section{Data Analysis}

Behavioral response times to the comparison decisions were analyzed using paired-samples $t$ tests among the semantic categories and between the semantic and control blocks. Neuroimaging data were preprocessed and analyzed using the Brain Voyager 2000 (Brain Innovation B.V.) software. Preprocessing steps included 6-parameter 3-D motion correction, slice-scan time correction using linear interpolation, voxel-wise linear detrending, and spatial smoothing with an 8-mm FWHM Gaussian kernel. Spatial normalization was performed using the standard 9-parameter Talairach landmark method (Talairach \& Tournoux, 1988). For analyses, a general linear model that included five regressors that modeled the BOLD response to each semantic condition and to the visuomotor control was defined for each participant. Each regressor was convolved with a standard gamma model (Boynton, Engel, Glover, \& Heeger, 1996) of the hemodynamic impulse response function. Following multiple regression from the general linear estimates of the conditions, first- and second-order random-effects analyses were conducted in line with our a priori predictions. To isolate candidate regions of interest in the first-order analysis, weighted contrasts were conducted for each category relative to the other semantic categories $(p<.01$, uncor- rected). For example, to localize regions that were preferentially active for body parts, this category was contrasted with the other three semantic object classes: birds, clothing, and fruits. Increased activation for the specific category was assessed in comparison with the control condition in paired sample $t$ tests. Candidate semantic regions in which the category of interest did not significantly differ $(p<.05)$ from the control condition were excluded from further analysis. Second-order orthogonal contrasts between the remaining categories were conducted to examine the category specificity of average block activity in each of these candidate semantic regions. Any regions that showed a significant difference $(p<.05$, Bonferroni correction) in the direct contrasts among the remaining semantic categories (e.g., among birds, clothing, fruits) rejected the category specificity hypothesis that only a category of interest (e.g., body parts) should show increased activation. These regions were instead classified as category-selective areas (i.e., two or more categories showed significant increased activation). That is, regions in which only one category showed increased activation are reported as category-specific areas (see Table 1). Areas in which activation increased for multiple categories are reported as category-selective areas (see Table 2).

Although the $p$ values of the first-order statistical parametric maps were not corrected for multiple voxel-wise comparisons, there are at least two reasons that explain why this threshold is warranted. First, the random-effects analysis took into account both the within- and between-subjects variance, allowing the results to be generalized to the population from which the sample was drawn and making the results statistically robust. In addition, the second-order analyses were based on variance within and between subjects from the summary condition data of each candidate semantic region. Areas not passing these second-order random-effects tests, with the statistical threshold Bonferroni corrected for multiple between-category comparisons, are not reported, to further reduce the chances of Type I errors.

\section{RESULTS}

The behavioral results indicated no significant differences in response times for the forced choice decisions between the visual $(M=883.10 \mathrm{msec}, S E=28.71 \mathrm{msec})$ and functional $(M=887.87 \mathrm{msec}, S E=20.46 \mathrm{msec})$ categories or between the individual categories (Birds: $M=$ $874.31 \mathrm{msec}, S E=31.88 \mathrm{msec}$; Body parts: $910.94 \mathrm{msec}$, $S E$ : 24.93 msec; Clothing: $M=864.80 \mathrm{msec}, S E=$ $15.99 \mathrm{msec}$; Fruits: $M=891.88 \mathrm{msec}, S E=25.54 \mathrm{msec}$ ). Therefore, any observed differences between semantic categories in the neuroimaging data are likely indicative of how this knowledge relies on specialized brain regions, as 
Table 2

Areas Showing Category-Selective Activation for Visual or Functional Categories

\begin{tabular}{|c|c|c|c|c|c|c|c|}
\hline \multirow[b]{2}{*}{ Category } & \multirow[b]{2}{*}{ Region } & \multirow[b]{2}{*}{$\mathrm{BA}$} & \multicolumn{3}{|c|}{$\begin{array}{c}\text { Talairach } \\
\text { Coordinates }\end{array}$} & \multirow{2}{*}{$\begin{array}{l}\text { Spatial } \\
\text { Extent } \\
\left(3-\mathrm{mm}^{3}\right. \\
\text { voxels })\end{array}$} & \multirow[b]{2}{*}{$t$ Value } \\
\hline & & & $x$ & $y$ & $z$ & & \\
\hline Visual & $\begin{array}{l}\text { Right lingual gyrus } \\
\text { Left parahippocampal gyrus }\end{array}$ & $\begin{array}{r}17 \\
19 / 37\end{array}$ & $\begin{array}{r}16 \\
-25\end{array}$ & $\begin{array}{l}-86 \\
-51\end{array}$ & $\begin{array}{r}-2 \\
-13\end{array}$ & $\begin{array}{r}8 \\
29\end{array}$ & $\begin{array}{l}3.28 \\
3.36\end{array}$ \\
\hline Functional & $\begin{array}{l}\text { Left inferior frontal gyrus } \\
\text { Left middle temporal gyrus }\end{array}$ & $\begin{array}{l}44 / 45 \\
21 / 37\end{array}$ & $\begin{array}{l}-50 \\
-51\end{array}$ & $\begin{array}{r}11 \\
-43\end{array}$ & $\begin{array}{r}13 \\
-3\end{array}$ & $\begin{array}{r}104 \\
18\end{array}$ & $\begin{array}{l}3.41 \\
3.62 \\
\end{array}$ \\
\hline
\end{tabular}

opposed to domain-general mechanisms. Forced choice responses took significantly longer $[t(12)=8.86, p<.001]$ : about 300 additional milliseconds, for the semantic categories $(M=885.48 \mathrm{msec}, S E=24.58 \mathrm{msec})$ than for the visuomotor control $(M=588.09 \mathrm{msec}, S E=29.09 \mathrm{msec})$.

The neuroimaging results indicate that cortical regions known to support the sensory encoding of object properties were activated when participants considered similarities between word items. In particular, category-specific analyses identified cortical regions that were active for only one of the four tested categories. This result was likely based on the role of these regions in supporting the sensory properties involved in perceptual experiences with the associated objects. Trials containing fruit items were specifically associated with increased activation in bilateral regions of the medial orbitofrontal cortex (Figure 2A) in contrast to baseline activity for the other three categories and the control condition (Figure 2B). Recent neuroimaging work suggests that these orbitofrontal areas are involved in representing distinct tastes (O'Doherty, Rolls, Francis, McGlone, \& Bowtell, 2001) and odors (Kareken et al., 2004), as does the convergence of gustatory and olfactory information (de Araujo, Rolls, Kringelbach, McGlone, \& Phillips, 2003).

Trials that required similarity judgments between body part labels activated the left lateral temporal occipitoparietal cortex (Figure 2C, Table 1), in contrast to trials for the other three categories (Figure 2D). This region is also activated when images of the human body are viewed (Beauchamp et al., 2002). A specific area in this general region, the extrastriate body area, is consistently found in individual participants, leading some investigators to suggest that this region is innately specified to perceptually process parts of the body (Downing et al., 2001). The prefrontal region, just anterior to the premotor cortex, may be specifically involved in the retrieval of action knowledge (Martin, Haxby, Lalonde, Wiggs, \& Ungerleider, 1995) associated with body parts. The set of regions specifically active for body parts showed robust activation and appeared to be part of a network of cortical regions involved in the perception of human actions and biological motion (Decety \& Grèzes, 1999; Grossman \& Blake, 2002).

Direct contrasts between the visual and functional categories indicate that semantic knowledge that is likely to rely on similar properties (as initially suggested by Warrington \& McCarthy, 1983) activates common brain regions. The seminal distinction between these categories was replicated, with the ventral temporal cortex associated with increased activity for the visual categories and the lat- eral occipitotemporal and frontal cortical regions exhibiting increased activity for the functional categories. That is, semantic categories that tend to preferentially rely on visual properties (i.e., fruits and birds) selectively activated a region in the parahippocampal gyrus of the left temporal lobe (Figure 3A, Table 2), whereas the functional categories were not associated with increased activity in this region (Figure 3B). This region of the ventral temporal cortex has been extensively associated with the visual properties of semantic knowledge in case studies (e.g., Warrington \& Shallice, 1984) and neuroimaging work (see, e.g., Martin et al., 1995), and has also been more generally associated with visual object recognition (Haxby et al., 2001). In particular, similarity judgments made on the basis of object color that specifically involve natural kinds (Thompson-Schill, Aguirre, D'Esposito, \& Farah, 1999) are associated with increased activity in the ventral temporal cortex (Mummery et al., 1998). Martin et al. (1995) found that object colors activated the ventral temporal cortex but that the generation of object actions activated regions in the middle temporal and prefrontal cortices.

Categories that tend to rely on functional properties (i.e., body parts and clothing) activated a set of regions in the left lateral temporal and prefrontal cortices. These effects appear to be driven by the network of regions specifically sensitive to body parts. However, an area just anterior to the extrastriate body area in the lateral temporal cortex (Figure 3C) also showed preferential activation for clothing stimuli, in contrast to the visual categories (Figure 3D). This result suggests that semantic knowledge for articles of clothing depends on areas associated with the perception and manipulation of body parts. These results are consistent with a recent meta-analysis of 24 studies by Grèzes and Decety (2001) that identified a network of cortical regions, including the observed prefrontal and lateral temporal areas. These areas are active not only for action knowledge retrieval, but also for action execution, observation, simulation, and verb generation. Indeed, the relationship between clothing and body parts appears to be much more pragmatic than simply getting dressed in the morning. Articles of clothing take their shape from the parts of the body on which they are worn; thus, knowledge of this semantic category appears to depend on regions that are specialized to process the human form.

\section{DISCUSSION}

The present results suggest that semantic decisions rely on multiple cortical areas, depending on the roles that those 
A

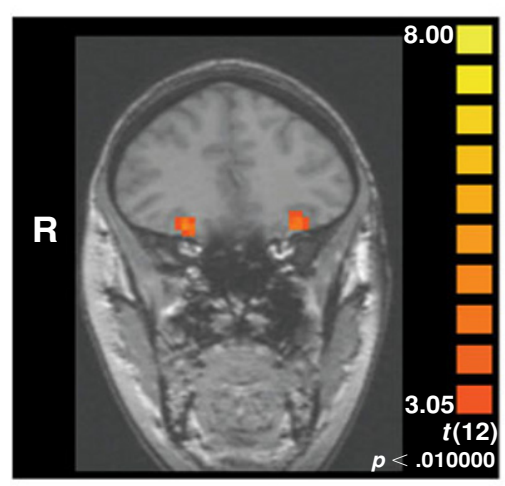

C

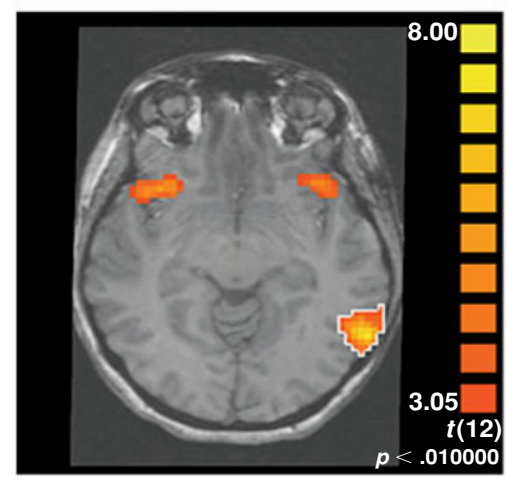

B

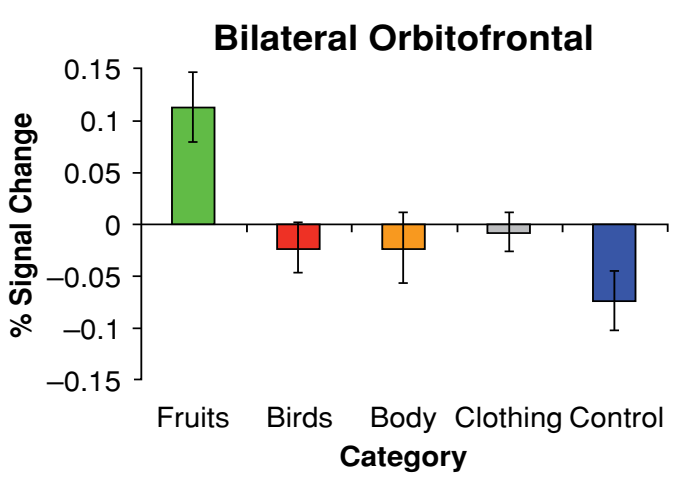

D

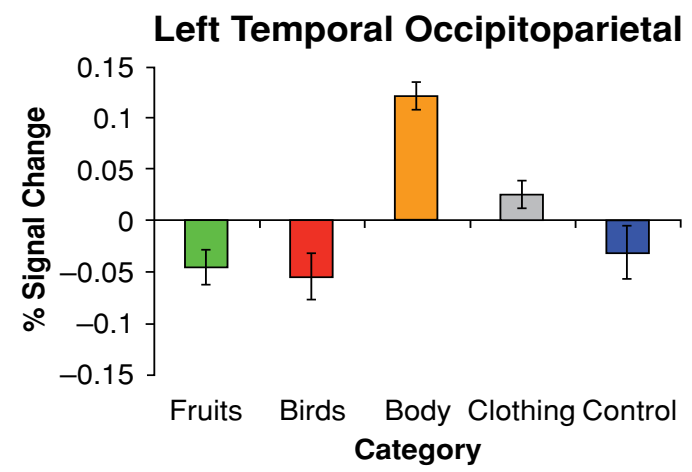

Figure 2. Category-specific regions implicated in mediating sensory processing. (A) Map of the $t$ statistic ( $p<.01$, uncorrected) for fruit items in bilateral orbitofrontal cortex. (B) Fruit specifically activated these regions, in contrast to the other categories. (C) Map of the $t$ statistic ( $p<.01$, uncorrected) for regions specifically active for labels of body parts, including the left temporal occipitoparietal cortex (outlined). (D) Body parts activate this region, in contrast to the other categories.

regions play in encoding perceptual and functional properties of objects. Multimodal semantic categories appear to activate multiple specialized regions, depending on the roles that sensory brain regions play in representing the perceptual properties of the referenced objects. Semantic categories that are likely to depend on unique types of object properties, such as flavor and biological motion, activate distinct cortical regions. In contrast, categories that rely on similar types of features, including visual or functional attributes, are associated with increased activation in common brain regions. These results suggest that similaritybased comparisons of word meanings evoke categorical distinctions in the patterns of cortical activation.

We found category-specific sensory regions for fruits and body parts, likely because of the roles of perceptual properties in distinguishing these semantic categories. In contrast, categories that were more likely to rely on either visual or functional properties were associated with selectively increased activity in regions that were previously implicated in representing these different types of object features by patient and other neuroimaging studies. In this regard, sensory mechanisms used to encode experiences with the given items also seem to represent the meaning of words and the superordinate categories to which they belong. Thus, semantic representations appear to depend on cortical activation patterns that are based on the constituent features involved. Categories that rely on separable types of features are likely to dissociate depending on the cortical regions involved. In contrast, categories that are similar in their reliance on one feature dimension are likely to depend on common cortical areas.

Orbitofrontal areas associated with the reward values for taste and smell (Rolls, 2000) and their integration (Small et al., 2004) were specifically activated by fruit stimuli. This region appears to support semantic decisions that require the flavor and reward properties of objects, even when photographs are presented (Simmons, Martin, $\&$ Barsalou, 2005). None of the other tested categories activated this region; however, it is important to note that none of the other categories could generally be considered to represent edible items. Since the activation of this region was likely driven by properties of taste and smell, other food items should similarly rely on this region. Indeed, we have recently found that this orbitofrontal region is specifically activated by the verification of a taste property, in contrast to other modalities (Goldberg, Perfetti, \& Schneider, 2006). A design more sensitive to individual trials could perhaps isolate activity in this region by utiliz- 
A

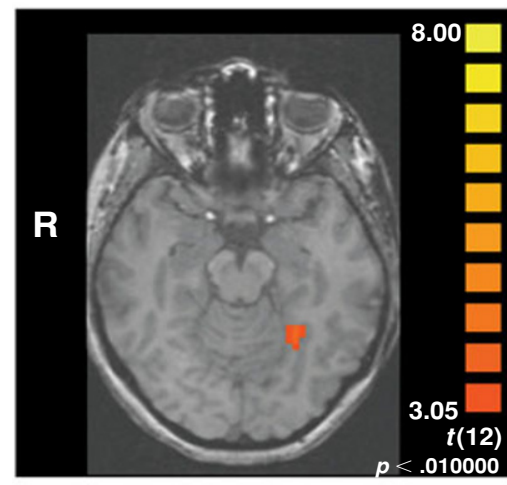

C

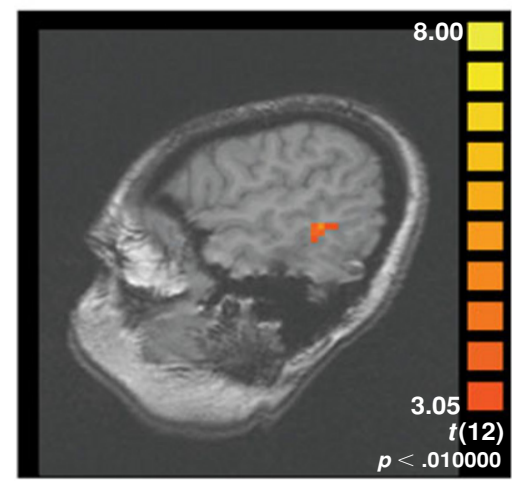

B

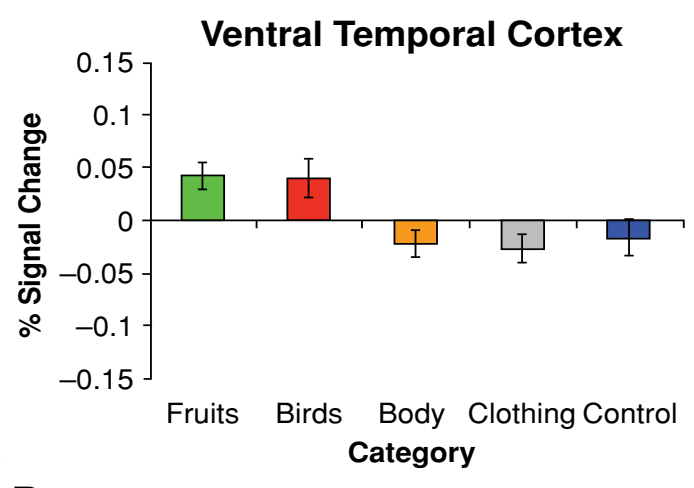

D

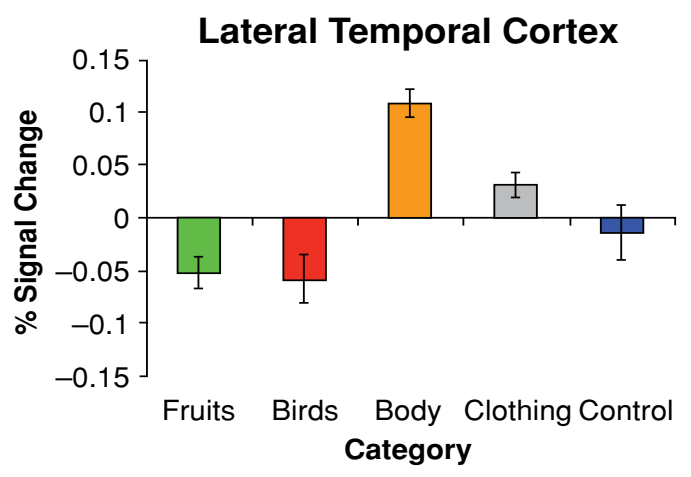

Figure 3. The reliance of visual and functional categories on sensory brain regions. (A) Map of the $t(p<.01$, uncorrected) for a visual semantic area in the left ventral temporal cortex. (B) This region is significantly activated for the visually biased and not the functionally biased categories. (C) Map of the $t$ statistic ( $p<.01$, uncorrected) for a left lateral temporal region showing greater activation for functional categories. (D) This region is responsive to body part names and clothing items, rather than to the visually biased categories.

ing categories that generally lack in such attributes (e.g., birds) but that contain specific instances of taste and smell properties (e.g., chicken and turkey).

Labels for body parts activated regions in the left lateral temporal occipitoparietal and prefrontal cortex that have previously been implicated in recognizing parts of the human body (Downing et al., 2001), in perceiving biological motion (Grèzes et al., 2001), and in accessing knowledge of actions (Tranel, Adolphs, Damasio, \& Damasio, 2001; Tranel, Kemmerer, Adolphs, Damasio, \& Damasio, 2003). These areas may be selectively involved in the representation of functional properties that are more necessary for some semantic categories than for others. In particular, a lateral temporal region that is most active for comparisons between body parts also showed increased activation to articles of clothing but not to more visually biased categories. In contrast, similarity-based comparisons within visual categories produced increased activity in the ventral temporal cortex, irrespective of the particular visual category. This dissociation between visually and functionally biased categories replicates effects that are apparent both in patients with focal brain damage and in neuroimaging studies with unimpaired individuals (Mar- tin \& Chao, 2001). In this regard, common brain regions appear to support object knowledge across semantic categories when decisions rely on similar types of properties.

Table 3 shows the pattern of activation associated with each of the tested categories. Some categories (i.e., birds and clothing) were associated with a single area of significant activation, whereas the other categories (i.e., fruits and body parts) were associated with multiple areas of increased activation. The cortical activation patterns provide a unique discrimination of each of the four categories. This result suggests that in accessing semantic knowledge, it might be possible to make a rapid response based on the overall set of areas activated. For example, nonoverlapping regions may be selectively used to discriminate among items that share many features in one perceptual modality, but differ significantly in others, such as determining whether a ball is a fruit. Comparisons of such semantic judgments that involve multimodal categories with distinct and common regions (e.g., fruits) could be tuned to operate in an area that is not typically shared with other items. In contrast, comparisons made between categories that rely on very similar types of features (e.g., fruit and vegetables) and - most likely — common cortical areas 
may lead to increased activation in each region in order to discriminate near neighbors (e.g., apple and tomato) in the semantic space.

These results suggest that other sensory brain regions should be activated when object knowledge is considered. Indeed, evidence is emerging that indicates sound judgments elicit selective activity in auditory association areas of the superior temporal cortex (James \& Gauthier, 2003; Kellenbach, Brett, \& Patterson, 2001). In addition, action words based on specific motor movements (e.g., lick and kick) activate areas directly adjacent to or overlapping with regions in the primary motor cortex that are associated with the actual body part movements (Hauk, Johnsrude, \& Pulvermüller, 2004). These findings indicate that semantic representations and the specific categories that rely upon them activate multiple sensory brain regions that encode visual, gustatory and olfactory, auditory, and motor experiences. Similarly, we (Goldberg et al., 2006) have found that the somatosensory cortex in the postcentral gyrus supports tactile judgments, in addition to primary and premotor regions. Specific categories may preferentially rely on each of these modality-based representations based on the diverse experiences one has with the indicated objects. For instance, object categories not tested (e.g., musical instruments) are likely to rely on a specific and predictable subset of perceptual properties, thereby selectively driving the associated regions of sensory cortex.

One potential difficulty with the present results is the limited number of categories that were examined. However, the generation-and-comparison paradigm is likely to yield robust patterns of activation for other categories that rely on similar conjunctions of properties. Distinguishing between similar categories may involve not only magnitude differences in distinct regions, but also voxel-wise patterns of correlated activity in the common areas, as suggested from visual object recognition (Haxby et al., 2001). Therefore, the differences between similar categories (e.g., fruit and vegetables) and perhaps even between specific items (e.g., peach and nectarine) may arise from variation in activity within the indicated sensory cortical regions.

While semantic categories seem to rely on sensory cortical regions, it is not clear how these disparate modalityspecific representations link together to give rise to the intuition of a holistic concept in memory. One possibility for such an integration of disparate elements of categorical knowledge may arise from multimodal convergence zones (A. R. Damasio, 1989; H. Damasio, Tranel, Grabowski, Adolphs, \& A. [R.] Damasio, 2004) or from how similar representations depend on a common cortical topography (Simmons \& Barsalou, 2003). Alternatively, sensory brain regions may be automatically activated whenever an object or its symbolic representation is presented (Martin et al., 1995). The representation of some objects may depend on the activation of related features (e.g., apple + green $=$ sour; apple + red $=$ sweet $)$ and is perhaps mediated by higher order areas (e.g., convergence zones) or by direct connections between sensory pathways. More passive tasks, such as identifying an item as a member of a given category, may not necessarily activate all of the modality-
Table 3

Distinct and Common Activations for the Tested Categories

\begin{tabular}{|c|c|c|c|c|}
\hline \multirow[b]{2}{*}{$\begin{array}{l}\text { Semantic } \\
\text { Category }\end{array}$} & \multicolumn{2}{|c|}{ Distinct ROIs } & \multicolumn{2}{|c|}{ Common ROIs } \\
\hline & Orbitofrontal & $\begin{array}{c}\text { Lateral } \\
\text { Frontotemporal }\end{array}$ & $\begin{array}{l}\text { Ventral } \\
\text { Temporal }\end{array}$ & $\begin{array}{c}\text { Lateral } \\
\text { Temporal }\end{array}$ \\
\hline Fruits & $\odot$ & & $\odot$ & \\
\hline Birds & & & $\odot$ & \\
\hline Body parts & & $\odot$ & & $\odot$ \\
\hline Clothing & & & & $\odot$ \\
\hline
\end{tabular}

specific properties involved in representing the category. For instance, identifying a peach as a fruit or naming a picture of the object could simply involve accessing prototypical visual properties that distinguish common fruits from other natural kinds. In a more demanding task, such as the similarity generation and comparison that we required, participants are expected to go beyond visual similarities to generate and select the best item while implicitly considering the other sensory properties associated with the items. By comparing cortical activation patterns across task contexts, it may be possible to determine whether certain properties are initially accessed with supplementary sensory features that are used to resolve a given semantic decision. It may also be possible to determine at which stage higher order representations, such as those theorized in convergence regions (A. R. Damasio, 1989; H. Damasio et al., 2004), become necessary to mediate conceptual comparisons.

In conclusion, the present results reveal a reliance on sensory brain regions when participants consider the similarities between object names. Semantic categories that rely on different types of perceptual properties activated distinct brain regions, whereas classes that depend on common sets of features activated common brain regions. These results support and extend feature-based views on the organization and wide distribution of semantic knowledge. The activation of cortical regions associated with taste and smell, visual processing, and biological motion indicates that object knowledge generally relies on multiple cortical regions associated with object perception, specifically when word items are used. The cortical distribution of multimodal semantic categories appears to reflect the feature differences and similarities apparent in the perceptual experiences with the associated objects.

\section{REFERENCES}

Barsalou, L. W. (1999). Perceptual symbol systems. Behavioral \& Brain Sciences, 22, 577-660.

Beauchamp, M. S., Lee, K. E., Haxby, J. V., \& Martin, A. (2002). Parallel visual motion processing streams for manipulable objects and human movements. Neuron, 34, 149-159.

Boynton, G. M., Engel, S. A., Glover, G. H., \& Heeger, D. J. (1996). Linear systems analysis of functional magnetic resonance imaging in human V1. Journal of Neuroscience, 16, 4207-4221.

Capitani, E., Laiacona, M., Mahon, B., \& Caramazza, A. (2003). What are the facts of semantic category-specific deficits? A critical review of the clinical evidence. Cognitive Neuropsychology, 20, 213-261.

CaramazzA, A., \& Mahon, B. Z. (2003). The organization of conceptual knowledge: The evidence from category-specific semantic deficits. Trends in Cognitive Sciences, 7, 354-361.

Caramazza, A., \& Shelton, J. R. (1998). Domain-specific knowledge 
systems in the brain: The animate-inanimate distinction. Journal of Cognitive Neuroscience, 10, 1-34.

Coltheart, M. (2001). Assumptions and methods in cognitive neuropsychology. In B. Rapp (Ed.), The handbook of cognitive neuropsychology: What defecits reveal about the human mind (pp. 3-21). New York: Psychology Press.

Damasio, A. R. (1989). Time-locked multiregional retroactivation: A systems-level proposal for the neural substrates of recall and recognition. Cognition, 33, 25-62.

Damasio, H., Tranel, D., Grabowski, T., Adolphs, R., \& DamaSIO, A. [R.] (2004). Neural systems behind word and concept retrieval. Cognition, 92, 179-229.

de Araujo, I. E. T., Rolls, E. T., Kringelbach, M. L., McGlone, F., \& PHILlips, N. (2003). Taste-olfactory convergence, and the representation of the pleasantness of flavour, in the human brain. European Journal of Neuroscience, 18, 2059-2068.

Decety, J., \& GRÈzes, J. (1999). Neural mechanisms subserving the perception of human actions. Trends in Cognitive Sciences, 3, 172-178.

Downing, P. E., Jiang, Y., Shuman, M., \& Kanwisher, N. (2001). A cortical area selective for visual processing of the human body. Science, 293, 2470-2473.

Forde, E. M. E., \& Humphreys, G. W. (2002). Category specificity in brain and mind. New York: Psychology Press.

Francis, W. N., \& KučERA, H. (1982). Frequency analysis of English usage. Boston: Houghton Mifflin.

Gainotti, G., \& Silveri, M. C. (1996). Cognitive and anatomical locus of lesion in a patient with a category-specific semantic impairment for living beings. Cognitive Neuropsychology, 13, 357-389.

GoldBerG, R. F. (2006). Unpublished data.

Goldberg, R. F., Perfetti, C. A., \& Schneider, W. (2006). Perceptual knowledge retrieval activates sensory brain regions. Journal of $\mathrm{Neu}$ roscience, 26, 4917-4921.

Grèzes, J., \& DeCETy, J. (2001). Functional anatomy of execution, mental simulation, observation, and verb generation of actions: A metaanalysis. Human Brain Mapping, 12, 1-19.

Grèzes, J., Fonlupt, P., Bertenthal, B., Delon-Martin, C., SegeBARTH, C., \& DeCETY, J. (2001). Does perception of biological motion rely on specific brain regions? NeuroImage, 13, 775-785.

Grossman, E. D., \& Blake, R. (2002). Brain areas active during visual perception of biological motion. Neuron, 35, 1167-1175.

HART, J., \& Gordon, B. (1992). Neural subsystems for object knowledge. Nature, 359, 60-64.

Hauk, O., Johnsrude, I., \& Pulvermüller, F. (2004). Somatotopic representation of action words in human motor and premotor cortex. Neuron, 41, 301-307.

Haxby, J. V., Gobbini, M. I., Furey, M. L., Ishai, A., Schouten, J. L., \& Pietrini, P. (2001). Distributed and overlapping representations of faces and objects in ventral temporal cortex. Science, 293, 2425-2430.

Hills, A. E., \& Caramazza, A. (1991). Mechanisms for accessing lexical representations for output: Evidence from a category-specific semantic deficit. Brain \& Language, 40, 106-144.

James, T. W., \& Gauthier, I. (2003). Auditory and action semantic features activate sensory-specific perceptual brain regions. Current Biology, 13, 1792-1796.

Kareken, D. A., Sabri, M., Radnovich, A. J., Claus, E., Foresman, B., Hector, D., \& Hutchins, G. D. (2004). Olfactory system activation from sniffing: Effects in piriform and orbitofrontal cortex. Neurolmage, 22, 456-465.

Kellenbach, M. L., Brett, M., \& Patterson, K. (2001). Large, colorful, or noisy? Attribute- and modality-specific activations during retrieval of perceptual attribute knowledge. Cognitive, Affective, \& Behavioral Neuroscience, 1, 207-221.

Landauer, T. K., \& Dumais, S. T. (1997). A solution to Plato's problem: The latent semantic analysis theory of acquisition, induction, and representation of knowledge. Psychological Review, 104, 211-240.

Margolis, E., \& Laurence, S. (1999). Concepts: Core readings. Cambridge, MA: MIT Press.

Martin, A., \& Caramazza, A. (2003). Neuropsychological and neuroimaging perspectives on conceptual knowledge: An introduction. Cognitive Neuropsychology, 20, 195-212.

Martin, A., \& Chao, L. L. (2001). Semantic memory and the brain: Structure and processes. Current Opinion in Neurobiology, 11, 194-201.
Martin, A., Haxby, J. V., Lalonde, F. M., Wiggs, C. L., \& UngerLEIDER, L. G. (1995). Discrete cortical regions associated with knowledge of color and knowledge of action. Science, 270, 102-105.

Martin, A., Wiggs, C. L., Ungerleider, L. G., \& Haxby, J. V. (1996). Neural correlates of category-specific knowledge. Nature, 379, 649652 .

Mummery, C. J., Patterson, K., Hodges, J. R., \& Price, C. J. (1998). Functional neuroanatomy of the semantic system: Divisible by what? Journal of Cognitive Neuroscience, 10, 766-777.

Mummery, C. J., Patterson, K., Hodges, J. R., \& Wise, R. J. (1996). Generating "tiger" as an animal name or a word beginning with T: Differences in brain activation. Proceedings of the Royal Society of London: Series B, 263, 989-995.

O’Doherty, J., Rolls, E., Francis, S., McGlone, F., \& Bowtell, R. (2001). The representation of pleasant and aversive taste in the human brain. Journal of Neurophysiology, 85, 1315-1321.

Perani, D., Cappa, S. F., Bettinardi, V., Bressi, S., Gorno Tempini, M., Matarrese, M., \& Fazio, F. (1995). Different neural systems for the recognition of animals and man-made tools. NeuroReport, 6, 16371641

Rolls, E. T. (2000). The orbitofrontal cortex and reward. Cerebral Cortex, 10, 284-294.

Rosch, E., Mervis, C. B., Gray, W. D., Johnson, D. M., \& BoyesBraem, P. (1976). Basic objects in natural categories. Cognitive Psychology, 8, 382-439.

Sacchett, C., \& Humphreys, G. W. (1992). Calling a squirrel a squirrel but a canoe a wigwam: A category-specific deficit for artefactual objects and body parts. Cognitive Neuropsychology, 9, 73-86.

SafFran, E. M., \& Schwartz, M. F. (1994). Of cabbages and things: Semantic memory from a neuropsychological perspective-A tutorial review. In C. Umiltà \& M. Moscovitch (Eds.), Attention and performance $X V$ : Conscious and nonconscious information processing (pp. 507-536). Cambridge, MA: MIT Press.

Simmons, W. K., \& BARSALOU, L. W. (2003). The similarity-in-topography principle: Reconciling theories of conceptual deficits. Cognitive Neuropsychology, 20, 451-486.

Simmons, W. K., Martin, A., \& Barsalou, L. W. (2005). Pictures of appetizing foods activate gustatory cortices for taste and reward. $\mathrm{Ce}$ rebral Cortex, 15, 1602-1608.

Small, D. M., Voss, J., Mak, Y. E., Simmons, K. B., Parrish, T., \& Gitelman, D. (2004). Experience-dependent neural integration of taste and smell in the human brain. Journal of Neurophysiology, 92, 1892-1903.

Solomon, K. O., \& Barsalou, L. W. (2001). Representing properties locally. Cognitive Psychology, 43, 129-169.

Stanfield, R. A., \& ZWAan, R. A. (2001). The effect of implied orientation derived from verbal context on picture recognition. Psychological Science, 12, 153-156.

TAlairach, J., \& Tournoux, P. (1988). A co-planar stereotaxic atlas of the human brain. New York: Thieme.

Thompson-Schill, S. L., Aguirre, G. K., D’Esposito, M., \& FARAH, M. J. (1999). A neural basis for category and modality specificity of semantic knowledge. Neuropsychologia, 37, 671-676.

Tranel, D., Adolphs, R., Damasio, H., \& Damasio, A. R. (2001). A neural basis for the retrieval of words for actions. Cognitive Neuropsychology, 18, 655-670.

Tranel, D., Kemmerer, D., Adolphs, R., Damasio, H., \& DamaSIO, A. R. (2003). Neural cocrrelates of conceptual knowledge for actions. Cognitive Neuropsychology, 20, 409-432.

Tyler, L. K., \& Moss, H. E. (2001). Towards a distributed account of conceptual knowledge. Trends in Cognitive Sciences, 5, 244-252.

WARrington, E. K., \& McCARThy, R. [A.] (1983). Category specific access dysphasia. Brain, 106, 859-878.

WARrington, E. K., \& MCCARThy, R. A. (1987). Categories of knowledge: Further fractionations and an attempted integration. Brain, 110, 1273-1296.

Warrington, E. K., \& Shallice, T. (1984). Category specific semantic impairments. Brain, 107, 829-854.

(Manuscript received July 29, 2005; revision accepted for publication March 13, 2006.) 